\title{
Texture Analysis of CT Images for Vascular Segmentation: A Revised Run Length Approach
}

\author{
Barbara Podda ${ }^{3}$ and Andrea Giachetti ${ }^{1,2}$ \\ 1 Universitá di Cagliari, Dip. Matematica e Informatica, \\ via Ospedale 72, 09124 Cagliari \\ giach@unica.it \\ 2 CRS4 - POLARIS, Loc. Piscinamanna 09010 Pula (CA), Italy \\ 3 DIEE, Universitá di Cagliari, Piazza d'Armi, 09123, Cagliari
}

\begin{abstract}
In this paper we present a textural feature analysis applied to a medical image segmentation problem where other methods fail, i.e. the localization of thrombotic tissue in the aorta. This problem is extremely relevant because many clinical applications are being developed for the computer assisted, image driven planning of vascular intervention, but standard segmentation techniques based on edges or gray level thresholding are not able to differentiate thrombus from surrounding tissues like vena, pancreas having similar HU average and noisy patterns [34]. Our work consisted in a deep analysis of the texture segmentation approaches used for CT scans, and on experimental tests performed to find out textural features that better discriminate between thrombus and other tissues. Found that some Run Length codes perform well both in literature and experiments, we tried to understand the reason of their success suggesting a revision of this approach with feature selection and the use of specifically thresholded Run Lengths that improves the discriminative power of measures reducing the computational cost.
\end{abstract}

\section{Introduction}

Digital diagnostic imaging modalities are providing the medical community with a large amount of high quality data that can be useful to obtain a better understanding of different pathologies. Computer assisted analysis of these images is fundamental to recover in a fast and objective way models, parameters and morphological descriptor of human organs. One of the most promising application of morphological reconstruction from digital images is the creation of patient specific vascular models from contrasted CT scans. Through the use of contrast media and helical multi-slice CT scanners it is possible to recover 3D models with sub-millimeter accuracy applying segmentation techniques based on data thresholding at specific HU values or more complex deformable models exploiting the HU level constancy of the contrast medium. Similar results cannot be obtained for the external walls of the vessel. In fact, especially in the aorta, it is possible to have deposits of thrombotic tissue between the blood and the vessel wall. These regions are not enhanced by the contrast liquid and their HU values are close to those of other tissues that can be found near the vessel. A few 


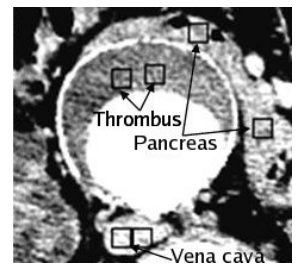

Fig. 1. Textures creating problems in aortic segmentation are thrombus, vena and pancreas, having close HU value averages

solutions to this problem have been proposed in literature. Olabarriaga et al. [3] trained a k-NN classifier to recognized vessel borders from samples taken on a line perpendicular to the vessel surface, Giachetti et al. 4] also analyzed 1D profiles giving ad hoc rules driving a deformable contour. Results are not always reliable, so that shape constraints have been also proposed ([14]) to avoid the inclusion in the vascular volume of voxels belonging to pancreas or vena cava. In this work we aimed at pointing out if this segmentation problem can be at least reduced by looking at texture descriptors. Two main questions have to be solved for this purpose: is there relevant information in the CT texture of the tissues of our interest? It is possible to find simple texture features that can be used in classical segmentation algorithms?

The paper is organized as follows: a short review of approaches for CT texture analysis is presented in Section 1; Section 2 presents an analysis of Thrombotic texture and a discussion about the meaning of the features based on Run Length Matrices, the most successful ones in this context. Section 3 presents experimental results supporting the analysis of the previous section and suggesting the possibility of designing ad hoc texture descriptors that can be used to improve thrombus segmentation.

\section{Texture Analysis and CT: A Review}

In recent years many studies based on texture analysis of CT images [501812] have been proposed to help the process of tissues and organs identification for medical applications. These studies suggest that some features, especially those based on Grey Level Run Length Matrix (GLRLM) are able to discriminate between different tissues types. Mir et al. 5] analyzed textural features based on Grey-Level Co-Occurrence Matrix (GLCM), Grey Level Run Length Matrix (GLRLM), and Grey Level Difference Matrix (GLDM). They used as input for the study CT Images of the abdomen with depth reduced to 256 levels and identified some features able to recognize the onset of disease in liver tissue which could not be identified even by trained human operators. In [9] Run Length Matrices are successfully applied to assess the spinal fracture risk from CT texture. Koss et al. [1] applied Haralick's Co-occurrence Matrix based features to classify abdominal tissues (not including those of our interest). They classified 
pixels on 6 texture classes using a neural network classifier, finding an accuracy of about $90 \%$. More recently, Raicu et al. 12 presented a texture dictionary for anatomical structures. They extracted features based on co-occurrence and run length matrices from CT Images. The features analyzed provided useful information about five different abdominal organs that could not be appreciated by human eye. In particular, High Grey Run Emphasis and Sum Mean proved to be the most discriminative indexes for the process of organs identification. In [8] the definition of Run Length Matrix is extended to the three dimensional space, in order to evaluate more properly textural features based on GLRLM for volumetric image data such e.g CT scans. They put emphasis on the fact that using many gray levels intensities, many runs would contain only one pixel, and consequently it is necessary to reduce the number of levels. They quantized the original 16 bit CT images into 32 gray-levels using linear mapping. The High Grey Level Run Emphasis and the Long Grey Level Run Emphasis descriptors proved to have a strong similarity for $2 \mathrm{D}$ and volumetric texture data. The usual approach in papers dealing with texture analysis is to compute a lot of features and give them as an input to a classifier. To reduce the dimensionality of the feature space Singular Value Decomposition or a more meaningful Linear Discriminative Analysis are used.9118. Our goal is slightly different, because we are not interested in building a classifier, but in computing one or a few features that could be inserted easily as constraints in a segmentation tool based, for example, in deformable surfaces or region growing. This is why we concentrated our efforts in feature selection and in understanding features' meaning for CT images of thrombus.

\section{Texture Analysis of CT}

Is CT texture meaningful? The problem of our interest is the discrimination of thrombus from surrounding tissues in abdominal CT. The main question is: can we distinguish between tissues that may be connected with the thrombotic region and the thrombus itself using texture information? A closer look at the histogram of thrombus (Fig. 2A) reveals that it is not Gaussian like that computed on a $\mathrm{CT}$ scan made with similar protocol on a phantom model of a material with constant density (Fig. 2B). Texture may therefore encode non trivial information about the tissue. This is not surprising, considering the literature results showing that Run Length Matrix based features are able to discriminate tissues on CT images. We are therefore interested in finding the reason why they are successful.

Run Length Matrices based features. RLM based features are computed in the following way: first the gray levels are subsampled in a coarser range of $\mathrm{N}$ values. Then runs of equal levels with lengths from 1 to $\mathrm{M}$ along a defined direction are computed. Runs are stored in a $\mathrm{MxN}$ matrix called Run Length Matrix and 11 values are usually derived from it: Short Run Emphasis (SRE), Long Run Emphasis (LRE), Gray Level Non Uniformity (GNU), Run Length Non Uniformity(RNU), Run Percentage (RPC), Low Gray Level Run Emphasis 

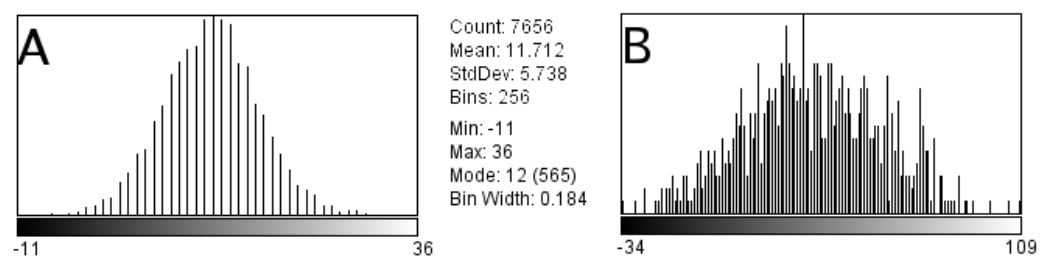

Count: 676

Mean: 34.430

StdDev: 24.797

Bins: 256

Min: -34

Max: 109

Mode: 31 (16)

Bin Width: 0.559

Fig. 2. While the histogram of the CT of a truly homogeneous region (left) is approximately Gaussian in detail, thrombus histogram (right) is, on a fine scale, complex, apparently coming from 2-3 overlapping distributions

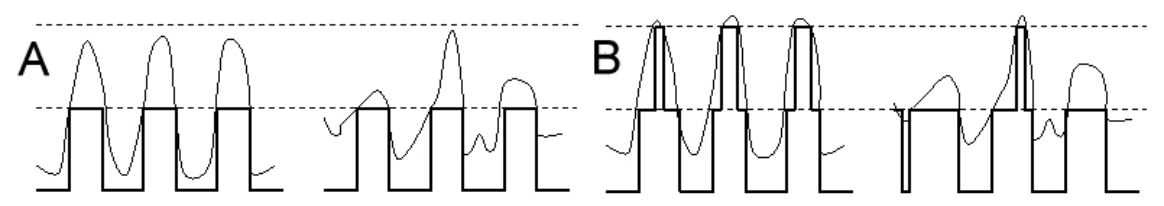

Fig. 3. Simple 1D example showing that just shifting a little the quantization limits, the gray level clustering used as a preprocessing before computing run length can miss (A) or capture (B) texture differences
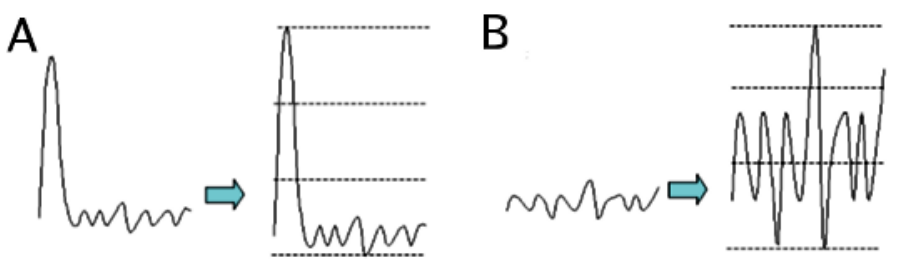

Fig. 4. If we reduce the original level range mapped into the reduced number of grays to the min-max interval, an outlier can differentiate two similar textures causing completely different runs $(\mathrm{A}, \mathrm{B})$

(LGRE), High Gray Level Run Emphasis (HGRE), Short Run Low Gray Level Run Emphasis (SRLGE), Short Run High Gray Level Run Emphasis (SRHGE), Long Run Low Gray Level Run Emphasis (LRLGE), Long Run High Gray Level Run Emphasis (LRHGE) (see [7/108]). If texture is not directional (as in our case), measures are usually averaged on all directions. Our hypothesis for the success of some RLM features on CT is that the texture is due to "grains" of different materials and sizes, and run lengths measure size and orientations of grains. With this interpretation, RLM methods can be interpreted as follows: the gray level subsampling acts as a "material classification" and the run length matrices measure statistical features of the classified regions.

The role of depth reduction. The critical step in the tissue discrimination is probably the pre-processing, i.e. the gray level subsampling performing the rough classification of the materials composing the image texels. This step is not, 
however, discussed in papers describing applications of the method. Without a gray level reduction, RLM is clearly not meaningful, being most of the runs equal to 1 . The matrix becomes zero everywhere except for the first column, containing the image histogram. In this limit, the few RLM-dependent features that appear meaningful in experiments converge to quadratic means, that can have discriminative power, but do not add new information to simple histogram based features. Clustering grays in a reduced number of levels, changes this result, making the discrimination obtained with some of the RLM features less correlated with histogram based measurements but still useful (see experimental section). Another fact to be considered is that the clustering function should not be a simple linear mapping of the full range or the min-max range of the region into the selected number of levels. In the first case, especially if we want to use a small number of gray levels, the clustering may miss meaningful structures, while in the second the effect of noise is too relevant and a single outlier pixel can change completely the transformed map, the corresponding homogeneous regions, the run lengths and the output of the texture descriptors. To understand these facts, let us consider the following situation: a 1D image, described by a continuous function (Fig.3). Run lengths computed at a coarse quantization, correspond to the length of the constant lines of the quantized functions. The coarse quantization in $\mathrm{A}$, creates the same runs from two completely different signals, and no features extracted from them could be used to discriminate the two texture. Different runs can be obtained not only with a finer quantization, but also with the same number of grays just taking quantization limits slightly shifted (B). Fig. 4 shows that for our application, a min-max rescaling of the gray levels before the quantization, performed in some applications before the gray level resampling, is not recommended: consider the $1 \mathrm{D}$ textures in $\mathrm{A}, \mathrm{B}$; they can be the same kind of tissue with just an outlier, or a small portion of another material on the left. The min-max transform creates two completely different signals that creates with the quantization limits of the dashed lines completely different runs. These considerations suggested us a new approach to compute gray level Run Length Matrices: knowing the HU value range and variations of the interested tissues we can create the low-depth image used for the feature computation clustering gray levels in a custom way, putting ad hoc thresholds, equal for all the ambiguous tissues. In this way it is possible to compute features from a very small number of levels (i.e. 4) without losing discriminative power. We therefore designed experiments aimed at selecting the ideal number of gray levels and the ideal thresholding function to extract the most powerful and efficient RLM features.

\section{Experimental Results}

To verify other authors' results, a comparative analysis of the textures was performed on classified image regions taken from 3 CT datasets of over 500 images acquired with a spiral CT scanner at resolution of about $0.6 \mathrm{x} 0.6 \mathrm{x} 2 \mathrm{~mm}$. We considered the three textures generating problems to usual segmentation algorithms 
Table 1. Intra and inter class Texture Features distances measured on Thrombus, Vena Cava and Pancreas from the original 12 bit images. Features considered are gray level mean, Co-Occurrence Matrix based (CM), Gray Level Difference Matrix based (CM) and Gray Level Run Length Matrix based (RLM).

\begin{tabular}{|l|c|c|c|c|c|c||c|c|c|c|c|c|}
\hline Distance & mean & CM1 & CM2 & CM3 & CM4 & CM5 & Distance & DM1 & DM2 & DM3 & DM4 & DM5 \\
\hline T-T & 0.24 & 0.85 & 0.85 & 0.06 & 1.06 & 0.13 & T-T & 0.13 & 0.20 & 0.08 & 0.10 & 0.51 \\
T-P & 2.77 & 0.33 & 0.31 & 0.65 & 2.01 & 0.08 & T-P & 0.08 & 0.75 & 0.35 & 0.44 & 0.91 \\
T-V & 5.89 & 0.11 & 0.11 & 1.89 & 0.59 & 0.25 & T-V & 0.25 & 1.67 & 1.42 & 1.44 & 1.65 \\
\hline
\end{tabular}

\begin{tabular}{|c|c|c|c|c|c|c|c|c|c|c|c|c|}
\hline D. & RLM1 & RLM2 & RLM3 & RLM4 & RLM5 & RLM6 & RLM7 & RLM8 & RLM9 & RLM10 & RLM11 \\
& SRE & LRE & GLNU & RLN & RPC & LGRE & HGRE & SRLGE & SRHGE & LRHGE & LRHGE \\
\hline TT & 0.25 & 0.32 & 0.19 & 0.20 & 0.20 & 0.24 & 0.23 & 0.15 & 0.27 & 0.39 & 0.08 \\
TP & 0.21 & 0.19 & 1.36 & 0.23 & 0.21 & 2.74 & 2.86 & 2.86 & 2.47 & 1.49 & 1.81 \\
TV & 0.05 & 0.05 & 1.39 & 0.05 & 0.00 & 5.79 & 5.93 & 5.75 & 5.27 & 2.74 & 3.45 \\
\hline
\end{tabular}

for the definition of thrombotic region limits, i.e. Thrombus itself, Vena Cava and Pancreas. The goal of the first series of experiments this was to find if there are measures different from the gray level average, that are sufficiently constant inside a class and different from the other classes. We considered the main texture features used in the papers reviewed in Section 1, i.e. features based on first order statistics, on Gray Level Co-Occurrence Matrices, Gray Level Difference Matrices and Run Length Matrices and tested all of them on the selected classified windows. We evaluated the discriminative power of features by measuring them on squared 10x10 windows correctly labeled as "Thrombus", "vena cava" and "pancreas". We computed texture features on these windows and their intra and inter-class averages and standard deviations. In detail, we computed on training sets of windows intra-class distance inside thrombus (T-T) and the inter-class distance between thrombus and pancreas (T-P) and thrombus and vena cava (T-V). The distance has been computed as follows: $D_{f}\left(X_{1}, X_{2}\right)=\left|F\left(X_{1}\right)-F\left(X_{2}\right)\right| / \sigma$ where $X_{1}$ and $X_{2}$ are two squares of $10 x 10$ pixels; $F\left(X_{1}\right)$ and $F\left(X_{2}\right)$ are the features $\mathrm{F}$ computed into $X_{1}$ and $X_{2} ; \sigma$ is the average standard deviation of the two classes. Table 1 shows the results obtained, showing clearly that gray level average and several measures derived by the Run Length Matrix (also Gray level Co-Occurrence Matrix and Gray Level Difference Matrix), are able to discriminate between textures of our interest in CT images.

But what about feature correlation and complexity? Complexity of GLRLM, GLCM and GLDM is very high if we work with 12 bit images (matrices with 4096 rows).In many classification approaches this problem is not analyzed because the goal is offline classification and the idea is, like in [10], to compute a lot of features and then combine them in a small and more significant set using Linear Discriminant Analysis or PCA. Our goal is, however, different, so we are interested in selecting one or few features simple, discriminating and not correlated with the average in order to improve the segmentation based on gray level. Tang (10), demonstrated also a strong correlation of classic RLM features on Broadtz textures. We already proved that if depth is relevant, runs tends to 1 and features are also strongly correlated with the average gray level. This is 


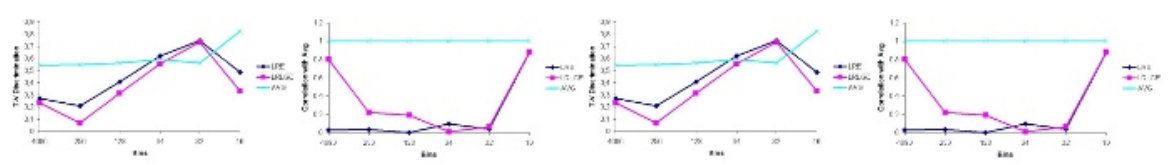

Fig. 5. Changes in features discriminative power and correlation with the (depth dependent) gray average due to bins subsampling. Features uncorrelated with average, like RLE still are uncorrelated and increase discriminative power up to 32 levels, then features lose their meaning. Using ad hoc thresholding, 8 levels are enough to preserve features discriminative power
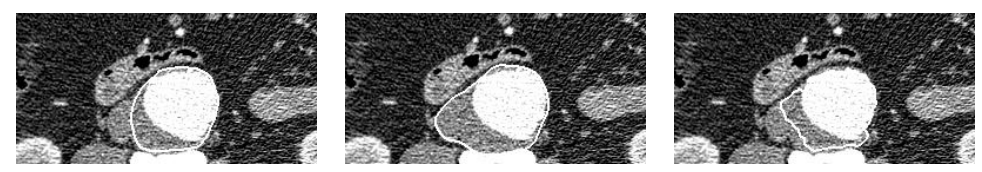

Fig. 6. Example of snake segmentation exploiting an image force computed in a $2 \mathrm{D}$ feature space. Left: original image with superimposed manually drawn thrombus contour. Center: result of snake based segmentation with image force based on gray level thresholding. Right: results of enhanced segmentation exploiting also a RLE feature (HGRE) map to constrain the snake.

confirmed by experimental data: RLM features showing discriminative power in Table 1 appear strongly correlated with the mean (coefficient close to 1 ). They do not seem to add relevant information (discrimination values are indeed quite similar). However, linearly decreasing the depth, we observed that graydependent features like LRLGE, SRHGE lose correlation with the mean and increase their discriminative power (see Fig 5, from data of the noisiest dataset available) and features, like LRE, having small discriminative power at original resolution, becomes clearly discriminative, maintaining their low correlation with the gray level average.

This behavior is evident up to 32 levels, with less performances are worse or correlation higher, probably because the correct thresholds for the texels differentiation are lost. But, even with just 8 levels, if threshold are selected ad hoc as suggested in the previous sections around the thrombus HU average, discriminative power of measures like LRE is preserved. This fact demonstrates that exist (a few) features very fast to be evaluated that are uncorrelated with the gray level average and add relevant discriminative power.

Many other tests will be necessary to better understand the features' behavior, it is however clear that texture features may be useful for thrombus delimitation and could be introduces also in fast vascular segmentation tool. As an example, let us use a feature to constrain deformable models. The idea is simple: image forces stopping balloon models inflation are computed in a feature space and do not depend only by local gray level values. An example of preliminary result of texture enhanced contour segmentation is shown in Fig. 5 . 
The application of texture filters in semi-automatic segmentation tools is however quite difficult because of problems in finding correct thresholds automatically and the low resolution and noise of texture descriptors maps.

\section{Discussion}

Texture analysis can improve the quality of the segmentation of ambiguous regions in CT images. Simple and effective features based on Run Length Matrices can be used in vascular segmentation when thrombotic tissue is not easily differentiated by surrounding tissues like vena cava. Our analysis show that the image depth reduction step, usually performed without an adequate discussion, has a great importance in determining the discriminative power of RLM based features. New tests will be performed on larger datasets to investigate feature behavior (also GLCM based) and the use of feature-space image forces in $2 \mathrm{D}$ and $3 \mathrm{D}$ deformable models will be investigated and tested.

\section{References}

1. M. de Bruijne et al, "Active shape model based segmentation of abdominal aortic aneurysms in CTA images". Proc. SPIE Medical Imaging 2002:Image Proc. 4684, 463-474 (2002)

2. M. Subasic, S. Loncaric, E. Sorantin, "3-D image analysis of abdominal aortic aneurysm", Proc. SPIE Medical Imaging vol. 4684, pp. 1681-1689, 2002

3. S.D. Olabarriaga, M. Breeuwer, W.J. Niessen, "Segmentation of Abdominal Aortic Aneurysms with a Non-parametric Appearance Model", LNCS 3117, ECCV Workshops CVAMIA and MMBIA, pp.257-268 (2004).

4. A.Giachetti and G.Zanetti, "AQUATICS Reconstruction Software: The Design of a Diagnostic Tool Based on Computer Vision Algorithms". LNCS 3117, ECCV Workshops CVAMIA and MMBIA, pp. 48-63 (2004).

5. A.H. Mir, M. Hanmandlu, S.N. Tandon, "Texture Analysis of CT Images", IEEE Engineering in Medicine and Biology, 14:6 pp. 781-786 (1995)

6. R.M. Haralick, K. Shanmugam, Its'Hak Dinstein, "Textural Features for Image Classification", IEEE Trans. on Systems, Man, and Cybernetics, 3,6:610-621(1973)

7. M.M. Galloway, "Texture Classification using grey level run lengths", Comp. Graphics and Image Processing, 4: 172-179 (1975)

8. D.H. Xu et al., "Run Lenght Encoding for Volumetric Texture", Proc. of the 4th IASTED Int. Conf. on Visualization, Imaging, and Image Processing, 2004.

9. M. Ito et al., "Trabecular texture analysis of CT images in the relationship with spinal fracture." Radiology. 1995 Jan;194(1):55-9

10. X, Tang, "Texture information in run-length matrices" IEEE Trans. on Image Processing, 7:11 , pp. 1602-1609 (1998)

11. J. E. Koss et al., "Abdominal Organ Segmentation Using Texture Transforms and a Hopfield Neural Network", IEEE Trans.on Medical Imaging, 18:7 (1999).

12. D. S. Raicu et al., "A Texture Dictionary for Human Organs Tissues' Classification", Proc. 8th World Multiconf. on Syst., Cyb. and Informatics, Orlando, USA (2004) 